\title{
A INFLUÊNCIA DA ADOÇÃO DE PRÁTICAS DE RESPONSABILIDADE SOCIAL CORPORATIVA NO COMPORTAMENTO DE COMPRA DA ALTA E BAIXA RENDA
}

\section{THE INFLUENCE OF CORPORATE SOCIAL RESPONSIBILITY PRACTICES ON THE LOW AND HIGH INCOME CONSUMER BEHAVIOR}

\section{Ronan Torres Quintão \\ CEFET-MG}

rtquintao@gmail.com

Giuliana Isabella

Faculdade de Economia, Administração e Contabilidade da Universidade de São Paulo giuliana.isabella@gmail.com

Submissão: $31 / 03 / 2015$

Aprovação: 18/11/2015 


\title{
RESUMO
}

Este artigo tem como objetivo identificar a influência da responsabilidade social corporativa (RSC) no comportamento de compra da alta e baixa renda em relação à percepção de benefício, valor, e intenção de compra do cliente, considerando um contexto onde a empresa socialmente responsável pratica um preço dez por cento maior do que a concorrência. Foram realizados dois experimentos para testar duas hipóteses sobre as relações da renda com o RSC. Como resultado verificou-se que ambos os grupos de consumidores de alta e baixa renda preferem produtos de empresas socialmente responsáveis quando o preço do produto está de acordo com nível de renda e gasto do consumidor. Entretanto os consumidores de baixa renda avaliam melhor comparado com os de alta renda os produtos das empresas que adotam práticas de RSC do que as que não adotam. Limitações do estudo e sugestões futuras são apresentadas no final do artigo.

PALAVRAS CHAVE: responsabilidade social corporativa, renda, comportamento do consumidor, preço, GEE

\begin{abstract}
This article aims to identify the influence of corporate social responsibility (CSR) in the purchase behaviour on high and low income people. The influences analysed are the perception of benefit, value, intent to purchase and attitude. We used a context of socially responsible company practice a price of ten percent higher than the non-socially responsible companies. We conducted two experiments to test two hypotheses. As a result, we found that both groups of high and low income consumer seek products which companies are socially responsible. Low-income consumers, when the product's price is compatible with their income, evaluate the products from CSR companies better compared to non-CSR companies than high-income consumers. Limitation of our study and future suggestions are presented at the end of the article.
\end{abstract}

KEYWORDS: social corporate responsibility, income, consumer behavior, price, GEE 


\section{INTRODUÇÃO}

A Responsabilidade Social Corporativa (RSC) emergiu como uma importante prática de mercado na década de 90 (HARRISON; FREEMAN, 1999; SEM; BHATTACHARYA, 2001). Com estudos sobre o assunto, as empresas perceberam que a adoção do comportamento socialmente responsável é necessário não apenas para responder às obrigações externas (leis e demandas dos stakeholders), mas também para aumentar a competitividade da empresa (SAEIDI; SOFIAN; SAEIDI; SAEIDI; SAAEIDI, 2015), reter funcionários (BARAKAT; ISABELLA, 2014) e melhorar o desempenho financeiro da empresa (CHENG; IOANNOU; SERAFEIM, 2014) no mercado. A partir de então, diversos pesquisadores começaram a estudar a RSC em conjunto com o comportamento do consumidor (VASCONCELOS; ALVES; PESQUEUX, 2012) para melhor entender a sua influência nas práticas de mercado e consumo.

Na perspectiva do marketing, os benefícios da adoção de práticas de RSC estão relacionados com uma avaliação positiva por parte do consumidor, influenciando suas crenças e atitudes em relação ao produto (FERREIRA; ÁVILA; FARIA, 2010) e à escolha e recomendação da marca (KLEIN; DAWAR, 2004; SEN; BHATTACHARYA, 2001). As pesquisas sobre RSC indicam que o consumidor percebe um benefício adicional na compra de produtos de empresas que adotam esta prática (BARONE; MIYAZAKI; TAYLOR, 2000; MOHR; WEBB, 2005; FERREIRA; ÁVILA; FARIA, 2010). Esta percepção da responsabilidade social influencia a imagem da marca e da empresa, a propensão do consumidor para comprar o produto e escolher o varejo, e o desempenho financeiro da empresa (LUO; BHATTACHARYA, 2006).

Entretanto, apesar de algumas pesquisas de mercado já sinalizarem a influência da variável renda na percepção das práticas de RSC adotas pelas empresas ainda que de forma descritiva, sem de fato entender o fenômeno sob um ponto de vista científico (exemplo: Instituto Akatu e Instituto Ethos, 2010), nenhum estudo acadêmico até então analisou o papel da variável renda no comportamento do comprador em relação à adoção de práticas de RSC pelas empresas.

A variável demográfica renda é uma das mais importantes (VAN KENHOVE; DE WULF, 2000). Ao lado do nível de educação, a renda é bastante utilizada na segmentação de consumidores (EDMONSON, 1995; NACHUM, 1994). A base da pirâmide compostas por 
pessoas de baixa renda, vem se tornando um importante e crescente mercado (BARKI; PARENTE, 2010) no Brasil e no mundo. Entretanto, as pesquisas realizadas até então não respondem à seguinte pergunta: tanto os consumidores de alta, quanto os de baixa renda pagam mais pelos produtos de empresas que adotam práticas de RSC? Eles percebem valor e benefício nestes produtos? Desta forma, o objetivo geral deste estudo é identificar a influência da renda do cliente na percepção de benefício, valor, intenção de compra diante da adoção de práticas de RSC pela empresa.

Analisar como agem os consumidores de diversas rendas significa contribuir no conhecimento do RSC e do comportamento do consumidor. O conhecimento gerado pelas respostas a perguntas de como as variáveis de marketing se relacionam, logicamente, levam a novas questões (HUBBARD; LINDSAY, 2002) e abrem possibilidades para a ampliação do campo de pesquisa. Este artigo está dividido em quatro partes: primeiro é apresentado um referencial teórico sobre RSC e renda, depois é explicado o primeiro e em seguida o segundo estudo empírico com suas respectivas análises. $\mathrm{O}$ artigo é finalizado com as considerações finais e a apresentação de sugestões de pesquisas futuras.

\section{RESPONSABILIDADE SOCIAL CORPORATIVA}

A adoção da RSC pelas empresas é uma prática antiga, desde o mercantilismo. O primeiro livro publicado sobre o tema foi de Bowen (1953): Social Responsibilities of the Businessman. Nessa época o conceito mais trabalhado era o de filantropia, que consistia na doação do patrimônio particular do dono da empresa e não do patrimônio da própria empresa. Ideias semelhantes foram defendidas por Friedman e Friedman (1962), que ainda hoje exercem forte influencia no pensamento capitalista, referente à relação da empresa com a sociedade. Para eles, as empresas, ao pagarem seus impostos, nada mais devem à sociedade, pois o objetivo empresarial é maximizar o retorno dos seus acionistas e não para a sociedade. O trabalho filantrópico deve apenas ser realizado por pessoas, e não pelas empresas. $\mathrm{Na}$ década de 1960, McGuire (1963) insere as empresas nessa discussão e argumenta que estas devem ter responsabilidades sociais além daquelas descritas na lei. Isto porque a RSC muitas vezes é definida como a obrigação da empresa em exercer um impacto positivo na sociedade e ao mesmo tempo minimizar o seu impacto negativo (PRICE; FERREL, 2006). 
Portanto, a orientação estratégica da empresa deve considerar a responsabilidade social em resposta às demandas da sociedade que se preocupa com o meio em que está inserido. De acordo com Vasconcelos, Alves e Pesqueux (2010, p.150) “as empresas, por meio de ações de RSC, vão muito além de apenas cumprirem as expectativas da sociedade, envolvendo-se diretamente em ações de regulação e na produção de bens públicos”. Porter e Kramer (2002) defendem ideias semelhantes, ao ressaltar que apenas quando os esforços e gastos com RSC produzirem tantos ganhos sociais, como benefícios econômicos é que se obtém um alinhamento e uma confluência entre os interesses dos acionistas e a filantropia corporativa. Nesse estágio é que as práticas da RSC assumem um papel estratégico, pois conseguem de forma mais plena influenciar a vantagem competitiva da empresa (PORTER; KRAMER, 2002).

Atualmente, as práticas de RSC buscam valorizar os funcionários e os direitos dos acionistas, mantendo uma boa relação com o meio ambiente, fornecedores, sociedade e clientes (BARAKAT; ISABELLA, 2014). A RSC se refere ao atendimento das expectativas do ambiente que a empresa está inserida em relação à questões econômicas, éticas e legais, entretanto Mascarenhas, Dias e Baptista (2015) afirmam que a RSC ainda "vem sendo enviesada pelos temas ambientais, em detrimento de visões mais complexas dos problemas a serem enfrentados". Devido ao grande número de agentes envolvidos diretamente ou indiretamente de práticas de RSC, os estudos nesta área são classificados em quatro grandes correntes teóricas (CRANE et al., 2008): (1) desempenho social corporativo: as ações socialmente responsáveis das empresas são construídas considerando-se as políticas, programas e resultados tangíveis na relação com a sociedade, (2) teoria dos acionistas: foco em aumentar a lucratividade dos acionistas por meio de ações socialmente corretas, (3) teoria dos stakeholders: o sucesso da empresa dependerá de como o gerenciamento dos interesses das pessoas envolvidas com a organização será conduzido e como isto a afetará, (4) cidadania corporativa: entende a empresa como uma parte da sociedade, devendo estar envolvida em atividades sociais, como a filantropia. Kraisornsuthasinee e Swierczek (2009) ressaltam que as empresas que adotam práticas de RSC devem buscar realizar não só iniciativas filantrópicas, que são atualmente as mais visadas pelas organizações.

PERCEPÇÃO DO CONSUMIDOR, RENDA E RSC

O valor percebido pelo consumidor diante de uma oferta é o resultado da comparação dos benefícios que ele ganha na troca realizada com a empresa e os sacrifícios (financeiros ou 
não) que percebe como sendo necessários (ZEITHAML, 1988). Várias pesquisas constataram que a RSC pode influenciar positivamente a percepção do consumidor (BARONE; MIYAZAKI; TAYLOR, 2000, MOHR; WEBB, 2005). Como o valor percebido de uma compra, ou de um produto em geral é um antecedente de intenção de compra, não é difícil entender que se o RSC influencia o valor percebido de um produto, influencia também a sua intenção de compra. O estudo de Ferreira, Ávila, Faria (2010), por exemplo, demonstrou que as práticas de RSC estão relacionadas com benefício e valor percebido e a intenção de compra dos consumidores. Todavia, há pesquisas que encontraram resultados divergentes em relação o comportamento de compra, indicando que a adoção de práticas de RSC não influenciam o comportamento de compra do consumidor. Carrigan e Attalla (2001), por exemplo, abordaram o público entre 18 a 25 anos separados por gênero e não encontraram esta influencia.

A pesquisa de mercado realizada pelo Instituto Akatu e Instituto Ethos (2010) coloca luz sob uma outra perspectiva até então não trabalhada pelos estudos acadêmicos no campo do comportamento de compra do consumidor. A pesquisa envolveu 800 mulheres e homens com mais de 15 anos de idade de todas as classes sociais e regiões do Brasil e encontrou diferenças significativas em termos de classe econômica em relação ao comportamento do consumidor e práticas de RSC. Segundo o trabalho realizado pelos Instituto Akatu e Instituto Ethos, as classes $\mathrm{C}$ e $\mathrm{D}$ adotam um comportamento menos consciente em relação à responsabilidade social do que as classes A e B. A variável socioeconômica renda se apresenta como possível moderadora do comportamento do consumidor em relação à adoção de práticas de RSC pelas empresas sendo necessário o estudo e entendimento de sua influência.

Dentre as variáveis de segmentação, as geográficas, demográficas e socioeconômicas são as mais comumente utilizadas (KONUS; VERHOEF; NESLIN, 2008). Elas são conhecidas como variáveis determinantes do comportamento do consumidor, pois os caracterizam como indivíduos e exercem grande influência em suas necessidades e em seu comportamento de compra. Dentre as variáveis socioeconômicas, a renda continua a ser uma das mais utilizadas e eficientes variáveis de segmentação para diferentes categorias de produtos e serviços (VAN KENHOVE; DE WULF, 2000), sendo que para produtos de consumo, a renda, ao lado do nível de educação, é uma das variáveis explanatórias mais importantes (NACHUM, 1994). De acordo com Brandão e Parente (2012), a renda tem sido proposta e testada em diversas pesquisas sobre comportamento do consumidor. 
Desta forma, busca-se identificar se a renda pode ser considerada um dos motivos para os diferentes achados na literatura. No Brasil, dois estudos se destacam em relação à definição dos critérios de classificação econômica da população: o critério Brasil e o estudo realizado por Neri (2010). O critério Brasil, elaborado pela ABEP (Associação Brasileira de Estudos Populacionais), classifica as pessoas de acordo com o potencial de consumo (acesso e o número de bens duráveis que a pessoa possui: TV, rádio, banheiro, automóvel, empregada mensalista, máquina de lavar, geladeira entre outros) e grau de instrução do chefe de família, atribuindo pontos para cada item que no final são utilizados para definir a classe econômica da pessoa. Já o estudo de Neri (2010) define os estratos da classe econômica da população brasileira segundo a renda absoluta das pessoas. Segundo Neri (2010) a classe D possui uma renda domiciliar total de todas as fontes de até $R \$ 1.126$, a classe $C$, de $R \$ 1.126$ até $R \$$ 4.854, a B, acima de R \$ 4.854. Entretanto, cabe ressaltar que, apesar de vários estudos já terem sido utilizados abordando as classes econômicas tanto no Brasil como no exterior, segundo Prado (2008), não há consenso sobre a definição exata da renda familiar da baixa renda e da alta renda.

Tendo como base a discussão da literatura sobre a influência (ou não) das práticas de RSC no comportamento de compra dos consumidores, além do impacto da renda no comportamento do consumidor, desenvolve-se a primeira hipótese deste estudo (H1).

\section{H1: há diferença entre os segmentos de ALTA e BAIXA renda em contextos de compra de produtos com prática de $\mathrm{RSC}$, em relação ao benefício percebido $\left(H 1_{\mathrm{A}}\right)$, valor percebido $\left(H 1_{B}\right)$ e intenção de compra $\left(H 1_{C}\right)$ do cliente.}

Considerando o preço de compra do produto, Creyer e Ross (1997) observaram que os consumidores estão dispostos a pagar mais por produtos de empresas que adotam práticas de RSC. Similar a eles, a pesquisa realizada por Mohr e Webb (2005) relacionou o preço do produto com as práticas de RSC adotadas pela empresa. Os resultados indicaram que os consumidores americanos valorizam a RSC e podem utilizá-la como critério para a decisão de compra, mesmo quando não há paridade de preço entre os produtos analisados no momento da compra. Entretanto, até então, na literatura (exemplo: Ferreira, Ávila e Faria, 2010), o preço utilizado em experimentos realizados com o consumidor sobre a influência de práticas de RSC não tem variado de acordo com o perfil dos informantes. Geralmente, adota-se um valor semelhante para todos independentemente da classe econômica e/ou social. Entretanto, 
no Brasil, a pesquisa de mercado realizada pela LatinPanel (2004) identificou a diferença do preço pago entre a alta e baixa renda em diversos tipos de produtos. A baixa renda paga $41 \%$ a menos pelos produtos da categoria de creme e loção em relação à alta renda, $47 \%$ pela de cereal tradicional, $47 \%$ pela de chá líquido para beber e $58 \%$ pela de detergente líquido para roupa.

Em relação à percepção de justiça de preço e poder de compra os consumidores se preocupam mais em pagar por preços honestos e justos do que apenas conseguir o preço mais baixo (CRAWFORD; MATHEWS, 2001; ISABELLA 2015). Relacionado ao preço, muitos pesquisadores afirmam que quando o consumidor percebe um preço alto, este pode julgá-lo como injusto e considerar desistir da compra ou reclamar a respeito. Um preço economicamente justo significa um adequado a suas expectativas (ISABELLA 2015). De acordo Maxwell (2002) a equidade social está definida como de acordo com as regras. Regras, neste caso, são os processos ou os resultados de preços. O conceito justiça tem dois aspectos principais: sociais e econômicos. Equidade econômica está relacionada com a teoria econômica que poderíamos extrapolar os aspectos de emoções. Desta forma, é importante que se adeqúe os preços dos produtos aos dois públicos (alto e baixa renda) minimizando a questão de injustiça de preço. Surge a necessidade de se verificar o comportamento do consumidor de baixa renda em relação à adoção de práticas de RSC pela empresa considerando preços mais adequados ao seu nível de renda e gasto. Desta forma, tem-se a segunda hipótese $(\mathrm{H} 2)$.

H2: a adoção de práticas de RSC pela empresa resulta em benefício percebido $\left(\mathrm{H} 2_{\mathrm{A}}\right)$, valor percebido $\left(H 2_{B}\right)$ e intenção de compra $\left(H 2_{c}\right)$ pelo cliente de BAIXA diferentes da ALTA renda quando o preço do produto estiver adequado ao seu nível de renda e gasto mensal.

\section{ESTUDO I}

$\mathrm{O}$ estudo 1 foi feito para testar a hipótese $\mathrm{H} 1_{\mathrm{A}}, \mathrm{H} 1_{\mathrm{b}}$ e $\mathrm{H} 1_{\mathrm{c}}$. $\mathrm{O}$ quase-experimento foi instrucional com manipulação na prática de RSC: presença e ausência (variável independente). Considera-se o estudo um quase experimento, pois os participantes foram separados de acordo com as suas condições de renda: alta e baixa. Desta forma, o design foi 2 (renda: alta e baixa) x 2 (práticas de RSC: presença e ausência), between subject. As variáveis 
dependentes mensuradas por escalas foram: benefício percebido, valor percebido e intenção de compra. A coleta de dados foi realizada com alunos matriculados no curso de extensão oferecidos para a comunidade (informantes de baixa renda) de uma instituição de ensino de Belo Horizonte e com alunos de graduação da mesma instituição (informantes de alta renda). Os questionários instrucionais foram impressos e aplicados em sala de aula de acordo com a autorização dos professores. As perguntas do questionário e a sua distribuição para os participantes em sala de aula foi realizada aleatoriamente. Os informantes classificados como participantes de alta renda responderam o mesmo questionário que os de baixa renda.

Para os participantes da baixa renda (técnico), foram entregues questionários instrucionais impressos. A entrega dos questionários aos participantes foi feita de forma aleatória, dentro de salas de aula definidas por conveniência. Os questionários foram aleatorizados tanto em suas condições quanto na ordem das perguntas dependentes, com o objetivo de minimizar possíveis relações entre as questões. No caso da alta renda o processo de coleta ocorreu em laboratórios. Os participantes acessaram o link na internet, onde a pesquisa estava disponível. O programa utilizado para a coleta de dados foi o Qualtrics (software que aleatoriza as condições e a ordem das respostas dependentes). Assim como na coleta da baixa renda, a definição das salas de aula foi por conveniência. Os questionários foram aplicados durante o horário de aula, mediante autorização dos professores. Embora a coleta de dados tenha sido distinta entre os grupos, todas as perguntas e instruções, seguiram as mesmas regras e textos. Ou seja, os alunos da alta renda também responderam as escalas adaptadas para a baixa renda. Com a baixa renda, a pesquisa não foi realizada no computador, pois, de acordo com os próprios professores, alguns alunos teriam dificuldade em utilizar a internet

Como manipulação, este primeiro estudo buscou seguir o experimento realizado por Ferreira, Ávila e Faria (2010). Por meio de um questionário impresso, os participantes foram expostos aleatoriamente a um dos cenários também utilizados no experimento da Ferreira, Ávila e Faria (2010): com RSC ou sem RSC de uma situação de compra de uma calça jeans (quadro 1). Esse produto foi escolhido por ser unissex e comprado tanto pelos consumidores de alta, quanto de baixa renda. No cenário sem prática de RSC, a calça jeans custava $\mathrm{R} \$ 100$ e no cenário com prática de RSC, $\mathrm{R} \$ 110$. Muitos estudos defendem que a adoção do RSC pela empresa permite um preço premium (HOMBERG et al., 2005; DU, BHATTACHARYA, SEN, 2007) podendo o consumidor pagar um pouco mais pelo produto. O valor de $\mathrm{R} \$ 110$ foi utilizado no experimento porque, segundo Ferreira, Ávila e Faria (2010), dez por cento é o 
“patamar sugerido pela literatura como razoável para que o consumidor perceba os preços como diferentes".

\section{Quadro 1: Cenários do estudo}

Sem RSC: cenário A

Imagine que você deseja comprar uma calça jeans (para você ), e vai ao shopping em busca de opções. Durante seu passeio pelo shopping, você experimenta diversas calças jeans. Ao final, você conclui que a calça da marca A e a da marca B, vendidas em lojas diferentes, foram as que você mais gostou. Você pretende comprar uma das calças, por isso deverá escolher entre a marca A ou B. As duas marcas são de boa qualidade, têm um design bonito e são confortáveis. Você gostou igualmente das duas marcas. A calça da marca A custa $\mathrm{R} \$ 100$, enquanto que a calça da marca B custa $\mathrm{R} \$ 110$.

Com RSC: cenário A + cenário $\mathrm{B}$

A empresa da calça jeans da marca B realiza atividades de responsabilidade social, investindo em programas sociais e na melhoria das condições de vida das pessoas, gerando empregos, pagando impostos, protegendo o meio ambiente e promovendo qualidade de vida para os seus funcionários.

As escalas adaptadas por Ferreira, Ávila e Faria (2010) de estudos anteriores seguindo orientações de Netemeyer et al. (2003) para medir Benefício Percebido, Valor Percebido e Intenção de compra, foram de 7 pontos, sendo apresentados os extremos (1: discordo fortemente e 7: concordo fortemente) e o ponto médio (4: não concordo, nem discordo). Ao todo foram apresentadas 10 afirmações referentes às variáveis dependentes, como descritas no Quadro 2.

\section{Quadro 2 - Escala Benefício Percebido, Valor Percebido e Intenção de Compra}

Benefício percebido: se eu comprar a calça jeans da marca $X$ vou me sentir bem comigo mesmo; comprar a calça jeans da marca $X$ me faria sentir que estou fazendo a coisa certa; se eu comprar a calça jeans da marca $X$ estarei me beneficiando; comprar a calça jeans da marca X me proporcionaria uma satisfação pessoal.

Valor percebido: se eu comprar a calça jeans da marca $\mathrm{X}$, estarei recebendo o que meu dinheiro vale; se eu comprar a calça jeans da marca $X$ eu acho que estarei recebendo um bom valor pelo dinheiro gasto; a calça jeans da marca $\mathrm{X}$ é uma compra que vale à pena, pois acho que seu preço é razoável.

Intenção de compra: eu estou disposto a comprar a calça jeans da marca X; a probabilidade de eu compara a calça jeans da marca $X$ é alta.; eu provavelmente vou comprar a calça jeans da marca X.

\section{ANÁLISE DOS DADOS}

A pesquisa foi realizada com uma amostra não-probabilística de 122 participantes, seguindo a sugestão de Hair et al. (1998) de se obter ao menos 30 sujeitos em cada célula de pesquisa do experimento (Tabela 1). Com relação à escolaridade da amostra 55\% possuem curso superior incompleto, sendo que destes, 61 foram classificados como consumidores de alta renda e 7 de baixa renda. Vinte e três por cento da amostra possui o ensino médio completo e todos foram 
classificados como baixa renda. Todos os participantes da pesquisa classificados como sendo de alta renda são alunos de graduação ou pós-graduado, confirmando as afirmações do SES (socialeconomic status - traduzida aqui por classe sócio econômica) que nas sociedades modernas é possível afirmar que as pessoas são classificadas por nível educacional, e a renda é consequência dessa educação (KAMAKURA, MAZZON, 2013). Nas análises foram consideradas apenas as extremidades das rendas familiares: alta renda, acima de $\mathrm{R} \$ 4.854$ (NERI, 2010) e baixa renda, valor até $\mathrm{R} \$ 2.991$, definido de acordo com os dados obtidos pela pesquisa, pois não há um consenso sobre esse valor na literatura (PRADO, 2008). A classe econômica intermediária (de $\mathrm{R}$ \$ 2.992 a $\mathrm{R}$ \$ 4.853) foi excluída da amostra para contrastar o máximo possível o nível de renda e melhor identificar o seu papel como discriminadora de possíveis diferenças de respostas.

Tabela 1: Amostra Estudo 1

\begin{tabular}{lcc}
\hline & Com RSC & Sem RSC \\
\hline Consumidor de Alta Renda & 30 & 32 \\
Consumidor de Baixa Renda & 30 & 30 \\
\hline
\end{tabular}

Para analisar as escalas utilizadas, realizou-se o alfa de Cronbach. Obteve-se os seguintes resultados: para Benefício Percebido: $\alpha=0.9164$ itens $(n=121)$; Valor Percebido: $\alpha=0.8793$ itens $(n=122)$ e Intenção de Compra: $\alpha=0.9273$ itens $(n=122)$. Seguindo os parâmetros propostos por Netmeyer et al. (2003) e Hair et al. (1998) é possível afirmar que os valores encontrados são satisfatórios e as escalas podem ser utilizadas. Além disso, para se testar os pressupostos da ANOVA ou do GLM foram realizados os testes de Levene (homogeneidade das variâncias). Os resultados de significância foram superiores a $p>=0,05$ comprovando que os dados são heterogêneos.

Antes de se testar a hipótese 1 foram comparadas as médias das respostas da amostra dos consumidores sem distinção de classe econômica do cenário com RSC com o cenário sem RSC, utilizando o teste ANOVA e intervalo de confiança de 95\%. Os consumidores participantes da amostra, independentemente de sua classe econômica, percebem maior benefício $(p \leq 0,05)$ e valor $(p \leq 0,05)$ e possuem maior intenção de compra $(p \leq 0,05)$ em relação ao produto pesquisado (calça jeans) quando a empresa adota práticas de RSC, confirmando, em um outro contexto, o resultado obtido por Ferreira, Ávila e Faria (2010). A tabela 2 apresenta os resultados. 
Tabela 2: Teste Hipóteses $\mathrm{H1}_{\mathrm{A}}, \mathrm{H} 1_{\mathrm{B}}$ e $\mathrm{H} 1_{\mathrm{C}}$

\begin{tabular}{cccccc}
\hline Variável Dependente & RSC & N & Média & Desvio-padrão & Teste \\
\hline \multirow{2}{*}{ Benefício Percebido } & Com & 60 & 4,70 & 1,66 & \multirow{2}{*}{$\mathrm{F}(121,1)=4,96 \mathrm{p}=0,028$} \\
& Sem & 62 & 3,97 & 1,92 & \multirow{2}{*}{$\mathrm{F}(121,1)=6,07 \mathrm{p}=0,015$} \\
\hline \multirow{2}{*}{ Valor Percebido } & Com & 60 & 4,56 & 1,66 & \\
& Sem & 62 & 3,79 & 1,75 & \\
\hline \multirow{2}{*}{ Intenção de Compra } & Com & 60 & 4,78 & 1,82 & \multirow{2}{*}{$\mathrm{F}(121,1)=8,92 \mathrm{p}=0,003$} \\
& Sem & 62 & 3,76 & 1,93 & \\
\hline
\end{tabular}

Em relação a hipótese 1, o primeiro ponto a ser verificado é a interação entre renda e RSC. Para isso foi rodado uma Análise de Modelo Geral Linear Univariado (GLM). Analisando o modelo com a variável dependente Benefício Percebido, verificou-se que a interação não é significante $(\mathrm{F}=0,115 ; \mathrm{p}=0,735)$. Ao analisarmos apenas a variável $\mathrm{RSC}$, confirmamos os resultados apresentados anteriormente pela a ANOVA, sendo significante $(F=4,715 ; p=0,032$, $\eta$ parcial $=0,038)$. O mesmo ocorre para a variável Valor Percebido. Não há significância na interação $(\mathrm{F}=0,073 ; \mathrm{p}=0,788)$, mas novamente se encontra diferença nas condições com e sem $\mathrm{RSC}(\mathrm{F}=6,014 ; \mathrm{p}=0,016 ; \eta$ parcial $=0,048)$. Em relação a variável Intenção de Compra, dados parecidos foram encontrados. Não há uma interação significante e apenas o RSC apareceu ser significante no modelo $(\mathrm{F}=8,896 ; \mathrm{p}=0,003 ; \eta$ parcial $=0,072)$. Como resultado, o teste não encontrou diferença significante na interação. Para aprofundar a análise, foram comparadas as avaliações das variáveis dependentes em relação a presença e ausência da prática de RSC de forma separada.

Ao separamos os segmentos alta e baixa renda, verificamos que para os consumidores de baixa renda da amostra o fato da empresa adotar práticas de RSC não aumenta o benefício e o valor percebido do produto oferecido por ela e nem a intenção de compra dos respondentes, pois não há diferença entre as médias do grupo sem RSC e com RSC em relação a essas variáveis dependentes ( $\mathrm{p}>0,05)$. A Tabela 3 apresenta as médias dos scores em cada variável e condição, assim como os resultados da ANOVA. Para os consumidores de alta renda ao se realizar uma ANOVA do cenário com RSC versus sem RSC, considerando um intervalo de confiança de 95\% verifica-se que quando a empresa adota práticas de RSC, os participantes da amostra percebem maior benefício e valor $(p=0,05)$ e maior intenção de compra $(p \leq 0,05)$ em relação ao produto pesquisado (calça jeans). Maiores detalhes são apresentados na Tabela 4. 
Tabela 3: Estudo com a Amostra da Baixa Renda

\begin{tabular}{cccccc}
\hline Variável Dependente & RSC & N & Média & Desvio-padrão & Teste \\
\hline \multirow{2}{*}{ Benefício Percebido } & Com & 30 & 4,86 & 1,76 & \multirow{2}{*}{$\mathrm{F}(58,1)=1,39 \mathrm{p}=0,24$} \\
& Sem & 29 & 4,26 & 2,13 & \\
\hline \multirow{2}{*}{ Valor Percebido } & Com & 30 & 4,60 & 2,02 & \multirow{2}{*}{$\mathrm{F}(58,1)=2,54 \mathrm{p}=0,11$} \\
& Sem & 29 & 3,75 & 2,09 & \\
\multirow{2}{*}{ Intenção de Compra } & Com & 30 & 4,68 & 1,98 & \multirow{2}{*}{$\mathrm{F}(58,1)=3,56 \mathrm{p}=0,06$} \\
& Sem & 29 & 3,66 & 2,16 & \\
\hline
\end{tabular}

Tabela 4: Estudo com a Amostra da Alta Renda

\begin{tabular}{|c|c|c|c|c|c|}
\hline Variável Dependente & RSC & $\mathrm{N}$ & Média & Desvio-padrão & Teste \\
\hline \multirow{2}{*}{ Benefício Percebido } & Com & 30 & 4,54 & 1,57 & \multirow{2}{*}{$F(62,1)=3.86 p=0,05$} \\
\hline & Sem & 33 & 3,72 & 1,72 & \\
\hline \multirow{2}{*}{ Valor Percebido } & Com & 30 & 4,52 & 1,25 & \multirow{2}{*}{$F(62,1)=3,99 p=0,05$} \\
\hline & Sem & 33 & 3,83 & 1,44 & \\
\hline \multirow{2}{*}{ Intenção de Compra } & Com & 30 & 4,88 & 1,67 & \multirow{2}{*}{$F(62,1)=5,68 p=0,02$} \\
\hline & Sem & 33 & 3,85 & 1,74 & \\
\hline
\end{tabular}

Embora não tenha sido encontrado uma interação entre renda e RSC, o que rejeita H1, as análises separadas trazem o indício de que o comportamento dos consumidores de alta e baixa renda podem ser diferentes em relação a prática de RSC adotadas pelas empresas, conforme sugerido por alguns autores como Ferreira, Ávila e Faria (2010). Para verificar essa questão, um segundo estudo foi realizado.

Com os resultados do ESTUDO I surgiu o seguinte questionamento: considerando o experimento realizado, por que não há diferença entre as médias das respostas em relação ao valor e benefício percebido e intenção de compra em relação à adoção ou não da RSC quando se compara as rendas (alta versus baixa), mas há diferença na baixa renda quando se compara a adoção de RSC ou não? Uma possível explicação pode ser o valor de R 100 da calça jeans utilizado no experimento. Esse preço pode ser alto para baixa renda, considerando o seu nível mensal de gasto com vestuário, e baixo para alta renda. Segundo a LatinPanel (2004), o gasto com vestuário é de $4,7 \%$ do valor da renda mensal domiciliar tanto para alta quanto para baixa renda. Considerando este valor, o gasto mensal da alta renda com vestuário seria de $R \$ 228$, para o valor mínimo da renda familiar: $\mathrm{R} \$ 4.854$, e o da baixa renda, de $\mathrm{R} \$ 140$, para o valor máximo da renda familiar: $\mathrm{R} \$ 2.991$. Diante dos resultados encontrados, foi realizado um segundo estudo considerando as hipóteses $\mathrm{H}_{2 \mathrm{~A}}, \mathrm{H}_{2 \mathrm{~B}}$ e $\mathrm{H}_{2 \mathrm{C}}$. $\mathrm{O}$ segundo estudo buscou explorar a diferença do preço nos produtos oferecidos para a alta e baixa renda, além de adaptar o texto de manipulação, tornando-o mais adequado à realidade da baixa renda, e incluir questões de check de manipulação. 


\section{ESTUDO II}

O objetivo do segundo estudo foi analisar a relação do preço dos produtos oferecidos aos participantes da alta e baixa renda. Para verificar essa questão do preço, no segundo estudo, foram realizados 2 pré-testes. Um para definir o preço pago pela alta e baixa renda, e um para alinhar nomenclaturas e textos a baixa renda. O primeiro pré-teste foi realizado com 96 alunos de alta e baixa renda da mesma instituição de ensino onde o segundo estudo seria realizado, com o objetivo de verificar o preço médio pago pelos estudantes de baixa e alta renda por uma calça jeans. Como resultado obtivemos que a baixa renda paga, em média, $\mathrm{R} \$$ 59,00 por uma calça jeans, enquanto que a alta renda, $\mathrm{R} \$ 102$. Estes valores estão alinhados com os descritos pela pesquisa da LatinPanel (2004).

Observa-se que o valor pago por uma calça jeans pelos respondentes da baixa renda da amostra é, em média, 33\% menor do que a alta renda, demonstrando que o valor de $\mathrm{R} \$ 100$ utilizado no primeiro experimento está mais relacionado com a realidade da alta renda do que da baixa. Para adequar o preço à realidade de compra dos diferentes grupos, no segundo estudo buscou-se trabalhar com preços distintos para a calça jeans: baixa renda, $\mathrm{R} \$ 60$ sem RSC, e R\$ 66, com RSC (10\% de aumento no valor), e alta renda, R\$ 100 sem RSC e R\$ 110 com RSC. Os valores foram arredondados para facilitar o entendimento dos respondentes.

Para reduzir a heterogeneidade da amostra em relação à formação, profissão, moradia e garantir a aleatoriedade dos respondentes com o intuito de minimizar a influência das variáveis estranhas no experimento, a coleta do segundo estudo foi realizada com alunos formados no segundo grau que estão cursando o técnico no período noturno (baixa renda) e com alunos do curso de graduação (alta renda) da mesma instituição de ensino situada em Belo Horizonte, Minas Gerais.

Para os participantes da baixa renda (técnico), foram entregues questionários instrucionais impressos. A entrega dos questionários aos participantes foi feita de forma aleatória, dentro de salas de aula definidas por conveniência. Os questionários foram aleatorizados tanto em suas condições quanto na ordem das perguntas dependentes, com o objetivo de minimizar possíveis relações entre as questões. No caso da alta renda o processo de coleta ocorreu em laboratórios. Os participantes acessaram o link na internet, onde a pesquisa estava disponível. O programa utilizado para a coleta de dados foi o Qualtrics (software que aleatoriza as 
condições e a ordem das respostas dependentes). Assim como na coleta da baixa renda, a definição das salas de aula foi por conveniência. Os questionários foram aplicados durante o horário de aula, mediante autorização dos professores. Embora a coleta de dados tenha sido distinta entre os grupos, todas as perguntas e instruções, seguiram as mesmas regras e textos. Ou seja, os alunos da alta renda também responderam as escalas adaptadas para a baixa renda. Com a baixa renda, a pesquisa não foi realizada no computador, pois, de acordo com os próprios professores, alguns alunos teriam dificuldade em utilizar a internet.

Com o objetivo de se verificar se o valor gasto influenciaria a intenção de compra de produtos com responsabilidade social, tanto na alta como na baixa renda, em alguns cenários do experimento a quantidade de calça jeans a ser comprada no experimento foi aumentada para três unidades, mantendo o preço unitário.

O segundo experimento possui um design misto: 2 (baixa e alta renda) x 2 (com RSC e sem RSC) x 2 (1 calça jeans e 3 calças jeans). Cada participante respondeu duas das quatro condições propostas: RSC e quantidade de calças jeans (within subject). Essas condições foram aleatórias, por exemplo, o participante pode ter respondido sobre a compra de uma calça com responsabilidade social e depois sobre a compra de três calças jeans sem responsabilidade social. A renda das pessoas não foi manipulada, mas sim agrupada diante das respostas dos participantes da pesquisa. Considerando o perfil dos alunos do curso técnico (baixa e média renda) e de graduação (média e alta renda), ambos do noturno, da instituição de ensino, foram aplicados os questionários com os preços relacionados à classe econômica. Por causa dessa separação baseada na escolaridade, alguns questionários respondidos foram desconsiderados. Apenas os questionários dentro da renda desejada foram utilizados na pesquisa. Em relação ao número de calças, optamos por fazê-lo para incluir a questão de aumento do preço do item, podendo assim verificar se o preço final (alto - de três calças) poderia influenciar nas variáveis dependentes.

\section{PRE TESTE}

Baseado no texto de manipulação do estudo 1, antes de rodarmos o estudo 2, buscou-se realizar um pré-teste. Em uma favela da zona sul de São Paulo, os pesquisadores deste artigo coletaram as percepções e dificuldades do público de baixa renda em responder a um questionário de responsabilidade social corporativa. Foram coletados insights de 23 adultos 
moradores da região. Com esta nova visão o texto instrucional manipulado foi modificado. Alteramos para facilitar o entendimento do texto o que significa dar acesso à informação para os participantes de menor nível educacional. Mesmo com a alteração, mantivemos os elementos da RSC apresentados no texto do primeiro estudo, seguindo a literatura sobre o tema, que considera como prática de RSC o investimento e preocupação da empresa em programas sociais, assim como na melhoria da qualidade de vida das pessoas, sejam funcionários, clientes ou pessoas da comunidade, na proteção do meio ambiente e na busca por um desenvolvimento econômico da região onde se encontra. Além do texto de manipulação, as perguntas sobre Valor Percebido, Benefício Percebido, e Intenção de Compra, também tiveram sua linguagem adaptadas. Ao aplicar o pré-teste verificou-se certa dificuldade dos participantes para responderem algumas questões da escala adaptada por Ferreira, Ávila e Faria (2010). Assim, embora a escala utilizada tenha sido a mesma, algumas palavras foram modificadas.

\section{ANÁLISE DOS DADOS}

Duzentas e doze pessoas participaram do segundo estudo, sendo que 211 responderam a pergunta sobre renda social. Com relação à alta renda, 93\% dos participantes são solteiros, 85\% moram em casa própria ou com os pais, 74 possuem ensino médio completo ou superior incompleto e há uma concentração dos participantes entre 17 e 23 anos. Já na baixa renda, 90\% dos participantes são solteiros, $82 \%$ moram em casa própria ou com os pais e $80 \%$ possuem ensino médio completo ou superior incompleto e a maioria dos participantes também possui de 17 a 23 anos. Esses dados mostram a similaridade dos grupos em relação às idades e moradia. A diferença encontrada é basicamente de escolaridade e renda. A Tabela 5 mostra a distribuição de renda desses grupos. Observa-se que em geral os participantes do curso técnico possuem uma renda menor do que os participantes de graduação, a ANOVA confirma essa diferença de renda entre os participantes de cada local $(\mathrm{F}(1,211)=14,238$; p_valor=0,01). Mesmo sabendo que o poder aquisitivo dos grupos é diferente, buscou-se trabalhar com as extremidades das rendas, embora os resultados da análise tenham sido semelhantes, sendo desconsiderados 55 participantes da pesquisa, que possuem renda de $\mathrm{R} \$ 2.991$ a $\mathrm{R} \$ 4.854$.

Tabela 5 - Faixa de Renda Familiar dos Participantes versus Grupos de Estudo

\begin{tabular}{ccccc}
\hline $\begin{array}{c}\text { Instituição } \\
\text { de Ensino }\end{array}$ & $\begin{array}{c}\text { Abaixo de } \\
\mathrm{R} \$ 2.990\end{array}$ & $\begin{array}{c}\text { De R } \$ 2.991 \mathrm{a} \\
\mathrm{R} \$ \mathrm{R} \$ 4.854\end{array}$ & $\begin{array}{c}\text { Acima de } \\
\mathrm{R} \$ 4854\end{array}$ & \multirow{2}{*}{ Total } \\
\hline Graduação & $42(32 \%)$ & 37 & $53(40 \%)$ & 132 \\
Técnico & $46(57.5 \%)$ & 18 & $16(20 \%)$ & 80 \\
\hline Total & 88 & 55 & 69 & 212 \\
\hline
\end{tabular}


As análises de Alpha de Cronbach mostram que a adaptação da escala não interferiu na definição das categorias: Valor Percebido $(\alpha=0,644, n=210,3$ itens), Benefício Percebido ( $\alpha=0,787, n=211,4$ itens) e Intenção de Compra ( $\alpha=0,898, n=210,3$ itens), de Ferreira, Ávila e Faria (2010. A confiabilidade da escala foi garantida, uma vez que os valores dos alfas de Cronbach foram maiores que 0,60, seguindo a orientação de Hair et al. (1998).

Foram considerados nas análises apenas aqueles participantes que responderam de forma adequada ao manipulation check. Essa checagem foi realizada por meio de duas perguntas inseridas no final do questionário: qual o produto foi solicitado que o respondente se imaginasse comprando e qual era o preço do produto A e B? Como as pessoas responderam duas vezes ao questionário (duas condições), o manipulation check foi realizado duas vezes, desta forma, caso o participante tenha acertado apenas a manipulação de uma das condições, apenas as respostas desta condição foram mantidas. Dos questionários respondidos, 176 foram da baixa renda (88 respondentes) e 138 da alta renda (69 respondentes). Destes, foram utilizados na pesquisa 286. Foram excluídos da análise 28 questionários, sendo 21 da baixa renda e 7 da alta renda. Isso ocorreu, pois, alguns participantes não lembraram produto utilizado na pesquisa ou seus respectivos valores.

A Tabela 6 apresenta os cruzamentos das médias entre as condições estudadas, assim como a quantidade de questionários respondidos. Testes de Levene foram realizados para verificar a homogeneidade das variâncias das amostras. Em todos os casos, esse pressuposto foi confirmado com p_valor $>0,05$.

Tabela 6- Variáveis Independentes e Dependentes: Médias dos Escores

\begin{tabular}{ccccccc}
\hline $\begin{array}{c}\text { Quantidade } \\
\text { de Calças }\end{array}$ & $\begin{array}{c}\text { Responsabilidade } \\
\text { Social }\end{array}$ & Renda & $\begin{array}{c}\text { Benefício } \\
\text { Percebido }\end{array}$ & $\begin{array}{c}\text { Valor } \\
\text { Percebido }\end{array}$ & $\begin{array}{c}\text { Intenção de } \\
\text { Compra }\end{array}$ & N \\
\hline Uma Calça & Com RSC & Baixa Renda & 3,9146 & 4,0121 & 4,3089 & 41 \\
& & Alta Renda & 4,4758 & 3,9409 & 4,3333 & 31 \\
& \multirow{2}{*}{ Sem RSC } & Baixa Renda & 2,3819 & 2,7222 & 2,7315 & 36 \\
& \multirow{2}{*}{ Total } & Alta Renda & 2,6389 & 2,9722 & 2,9352 & 36 \\
& & Baixa Renda & 3,1981 & 3,409 & 3,5714 & 77 \\
& Com RSC & Alta Renda & 3,4888 & 3,4204 & 3,5821 & 67 \\
\hline \multirow{2}{*}{ 3 Calças } & Baixa Renda & 3,6118 & 3,3659 & 3,2927 & 41 \\
& \multirow{2}{*}{ Sem RSC } & Alta Renda & 3,8359 & 3,8854 & 3,7500 & 32 \\
& \multirow{2}{*}{ Total } & Baixa Renda & 2,7297 & 3,0000 & 2,6216 & 37 \\
& & Alta Renda & 2,4687 & 2,8125 & 2,7604 & 32 \\
& Baixa Renda & 3,1934 & 3,1923 & 2,9744 & 78 \\
& Com RSC & Alta Renda & 3,1523 & 3,3490 & 3,2552 & 64 \\
\hline Total & Baixa Renda & 3,7632 & 3,6890 & 3,8008 & 82 \\
& & Alta Renda & 4,1508 & 3,9127 & 4,0370 & 63 \\
& & Total & 3,9316 & 3,7862 & 3,9034 & 145
\end{tabular}




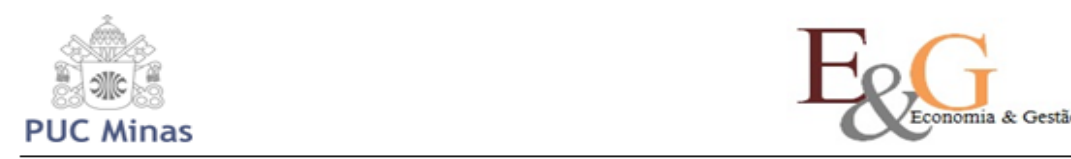

\section{ISSN 1984-6606}

\begin{tabular}{cccccc} 
Sem RSC & Baixa Renda & 2,5582 & 2,8630 & 2,6758 & 73 \\
& Alta Renda & 2,5588 & 2,8971 & 2,8529 & 68 \\
& Total & 2,5585 & 2,8794 & 2,7612 & 141 \\
\multirow{3}{*}{ Total } & Baixa Renda & 3,1957 & 3,3000 & 3,2710 & 155 \\
& Alta Renda & 3,3244 & 3,3855 & 3,4224 & 131 \\
& Total & 3,2547 & 3,3391 & 3,3403 & 286 \\
\hline
\end{tabular}

Para a análise dos dados foi utilizado uma equação de estimação generalizada mais conhecida como GEE (Generalized Estimating Equation). O GEE utiliza um modelo linear generalizado para estimar parâmetros de regressão e permite uma análise de dados longitudinais (BALLINGER, 2004). Dados longitudinais são caracterizados pelo fato de observações repetidas, para um assunto, tendem a estar correlacionadas (ZEGER, LIANG, ALBERT, 1988), exatamente o que pode ter ocorrido nesse estudo. Os participantes podem ter sofrido uma interferência ao responderem à segunda condição, já que tiveram um certo conhecimento prévio do assunto ao responderem à primeira condição. Nesse estudo utilizou-se a estimativa de máxima verossimilhança com função linear e estrutura de matriz de trabalho não estruturada, que, geralmente, é a mais adequada para quando não existe um padrão de auto correlação da variável dependente. Em todas os modelos apresentados a seguir, a resposta da afirmativa "é um problema para mim escolher calças jeans" do questionário foi utilizada como uma covariável.

O primeiro modelo rodado foi com a variável dependente Benefício Percebido. O modelo rodado foi fatorial com as três condições presentes. Este apresentou um ajuste com o valor do indicador QICC (Corrected Quasi Likelihood under Independence Model Criterion) de 1,029. Analisando a interação responsabilidade social e renda, obtivemos um Wald Qui Quadrado, que mede a eficiência das estimativas dos coeficientes da regressão original em satisfazer as restrições da hipótese nula, de 3.080 e p_valor de 0,079, não sendo significativo com 95\% de confiança. Entretanto, a condição responsabilidade social foi estatisticamente significante com Wald Qui quadrado de 65,458 e p_valor de 0,000. Para verificar a $\mathrm{H} 2{ }_{\mathrm{A}}$, separamos apenas a baixa renda; como resultado obtivemos um Wald Qui Quadrado de 28,995 e um p_valor de 0,000. Esses resultados suportam as hipóteses $\mathrm{H} 1_{\mathrm{A}}$ e $\mathrm{H} 2_{\mathrm{A}}$.

O segundo modelo analisado foi do Valor Percebido. O QICC do modelo foi de 1,880. A interação responsabilidade social, número de calças e renda não foi significante a $95 \%$ de confiança. O Wald Qui Quadrado dessa interação foi de 2,228 e o p_valor de 0,136. Nesse modelo apenas a responsabilidade social foi significante com Wald Qui Quadrado de 43.177 e 
p-valor de 0,000. Esses resultados suportam a hipótese $\mathrm{H} 1_{\mathrm{B}}$. Para analisar $\mathrm{H} 2_{\mathrm{B}}$, estudou-se apenas a amostra da baixa renda. Como resultado, obteve-se um Wald Qui Quadrado de 15,495 e p-valor de 0,000, suportando esta hipótese.

O terceiro modelo rodado foi o da Intenção de Compra. O QICC do modelo foi de 1,218. Analisando o modelo, observou-se que há uma interação entre o número de calças compradas e a responsabilidade social. O Wald Qui Quadrado dessa interação foi de 3,850 e o p_valor de 0,050. Quando os participantes de baixa renda analisaram a compra por uma calça, tiveram uma intenção de compra de 3,064 (escala de 1 a 7 pontos), ao comprar três calças a intenção foi de 2,943. Para os participantes da alta renda, a intenção de compra para uma calça é de 3,307 enquanto que para três calças foi de 3,029. As médias demonstram que tanto na alta renda como para a baixa renda, a quantidade de calças compradas altera a intenção de compra. Em relação a responsabilidade social, também foi encontrado uma diferença estatisticamente significante (Wald $=41,557$, p_valor=0,000). Não houve, entretanto, uma interação com a renda, o que significa que não há diferenças entre as rendas quando pensamos em produtos vindos de empresas socialmente responsáveis, o que confirma a hipótese $\mathrm{H} 1_{\mathrm{C}}$. Ao estudarmos apenas o grupo da baixa renda $\left(\mathrm{H} 2_{\mathrm{C}}\right)$, confirmamos que o número de calças é significante (Wald $=5,946$ p_valor $=0,015$ ) e que as pessoas preferem produtos onde as empresas são socialmente responsáveis (Wald $=22,568$, p_valor $=0,000$ ).

\section{CONSIDERAÇÕES FINAIS}

Na literatura, até então, não havia sido realizado estudos verificando a influência da variável renda no comportamento do consumidor em relação à adoção de práticas de RSC pelas empresas. Como contribuição teórica, este estudo buscou melhor entender a influência desta variável diante das características específicas de consumo da alta e da baixa renda ampliando o campo de conhecimento sobre comportamento do consumidor e Responsabilidade Social Corporativa. No primeiro estudo verificado se haveria percepções diferentes entre os consumidores de baixa e alta renda em relação à adoção de práticas de RSC. Considerando o valor de R\$ 100 para a calça jeans utilizada no experimento, os respondentes de baixa renda não perceberam maior ou menor benefício e valor, ou tiveram maior ou menor intenção de compra para o produto que adotava práticas de RSC em relação aos respondentes de alta renda. Os resultados do primeiro estudo da pesquisa revelaram, em relação à rejeição da primeira hipótese $\left(\mathrm{H}^{* *}\right.$, que a adoção de práticas de RSC aumenta a percepção de benefício e 
de valor dos consumidores em relação ao produto, assim como a intenção de compra, em relação ao cenário sem RSC, confirmando o resultado também obtido por Ferreira, Ávila e Faria (2010), Creyer e Ross (1997), Barone, Miyazaki e Taylor (2010), Mohr e Webb (2005), independentemente da renda da pessoa, não confirmando, entretanto, os achados descritos o estudo realizado pela LatinPanel (2004).

Com a intenção de se agregar novos achados ao conhecimento construído até então, foi verificado, no segundo estudo, se o preço utilizado na manipulação do experimento de alguma forma poderia influenciar as respostas dos informantes. Estudos como o realizado por Ferreira, Ávila e Faria (2010) não verificam especificamente esta questão (verificar o nível de aderência do produto à realidade do participante da pesquisa), que, segundo Crawford, Matheus (2001) e Isabella (2015), são fundamentais sob o ponto de vista do comportamento do consumidor. Por isso, o segundo estudo identificou e alterou os preços dos produtos de acordo com o nível de renda e gasto mensal dos respondentes da baixa renda, com o objetivo de se estender o entendimento sobre comportamento de compra dos consumidores pertencentes à classificação desta classe econômica. O preço pago, em média, por uma calça jeans pela baixa renda encontrado no estudo 2 foi de $R \$ 59$ e pela alta renda, $R \$ 102$, sendo estes valores alinhados com os resultados obtidos pela Latin Panel (2004) em relação à diferença do gasto com vestuários entre ambas as classes.

Melhor ajustando os valores trabalhados nos experimentos para adequar às duas classes econômicas (alta e baixa renda), verificou-se que a baixa renda percebe maior benefício, valor e possui maior intenção de compra de produtos que adotam práticas de RSC, sendo, neste novo estudo, aceitas as hipóteses $\mathrm{H} 2_{\mathrm{A}}, \mathrm{H} 2_{\mathrm{B}}$ e $\mathrm{H} 2_{\mathrm{C}}$. Este resultado amplia o conhecimento no campo acadêmico sobre comportamento do consumidor de baixa renda. Até então, nenhuma pesquisa acadêmica havia identificado que os consumidores de baixa renda percebem maior valor e benéfico, além de intenção de compra de produtos que adotam práticas de RSC. Na literatura tanto científica quanto de mercado pesquisada, apenas o trabalho do Instituto Akatu e Instituto Ethos (2010) encontrou diferenças significativas em relação à renda considerando o comportamento de compra e a adoção de praticas de RSC pelas empresas. Entretanto, diferentemente dos resultados obtidos neste artigo, segundo a pesquisa do Instituto Akatu e Instituto Ethos, a baixa renda adota um comportamento menos consciente em relação à responsabilidade social do que alta renda. $\mathrm{O}$ resultado obtido por este trabalho ao final dos 2 
experimentos realizado ajuda a colocar luz sob essa questão tanto no campo acadêmico quanto no campo corporativo.

O estudo contribui para o campo teórico ajudando a entender a influência do impacto da adoção da RSC pelas empresas na percepção dos consumidores de alta e baixa renda, ampliando os resultados das pesquisas realizadas sobre o tema até então. No campo empírico, o estudo reforça a importância da adoção de práticas de RSC e ao mesmo tempo revela a necessidade de melhor se conhecer o perfil dos clientes atendidos e suas necessidades. Como pesquisas futuras sugere-se a abordagem de experimentos de campo, trabalhando com o comportamento propriamente e não com a intenção de compra. Outros produtos, serviços podem ser explorados. Além de outros segmentos, como personalidade ou idade. 


\section{REFERÊNCIAS}

BALLINGER G. A. Using generalized estimating equations for longitudinal data analysis. Organizational Research Methods, v.7, n.2, p.127-150, 2004.

BARAKAT, S. R.; ISABELLA, G. Estudo sobre a relação entre desempenho social corporativo e retenção e satisfação de funcionários. XVII SEMEAD - Seminário em Administração, São Paulo, SP, ISSN:2177-3866, 2014.

BARKI, E.; PARENTE, J. Consumer Behavior of the Bases of the Pyramid Market in Brazil. Greener Management International, v. 56, p.11-23, 2010.

BARONE, M. J.; MIYAZAKI A.D.; TAYLORK.A. The Influence of Cause-Related Marketing on Consumer Choice: Does One Good Turn Deserve Another? Journal of the Academy of Marketing Science, v. 28, n.2, p. 248-262, 2000.

BERENS, G., VAN RIEL, C. B. M., \&VAN BRUGGEN, G. H. Corporate associations and consumer product responses: The moderating role of corporate brand dominance. Journal of Marketing, n.69, v.3, p.35-48, 2005.

BHATTACHARYA, C.B.; SEN S. Consumer-Company Identification: A Framework for Understanding Consumers' Relationships with Companies. Journal of Marketing, v. 67 p.7688, 2003.

BHATTACHARYA C.B.; SEN S. Doing Better at Doing at Good: When, Why, and How Consumers Respond to Corporate Social Initiatives. California Management Review, v. 47, n.1, p. 9-24, 2004.

BHATTACHARYA, S. S.; SAHAY, A., ARORA, A. P.; CHATUVERDI, A. A toolkit for designing firm level strategic corporate social responsibility (CSR) initiatives. Social Responsibility Journal, v.4, n.3, p.265-282, 2008.

BOWEN, H. R. Social responsibilities of the businessman. New York: Harper \& Row, 1953.

BRANDÃO, M. M.; PARENTE, J. Brasileiro gosta de muvuca? Impacto da densidade humana no comportamento de compra. Revista de Adminstração de Empresas, v.52, n.6, p613-627, 2012.

CARRIGAN, M., ATTALlA, A. The Myth of the Ethical Consumer - Do Ethics Matter in Purchase Behavior? Journal of Consumer Marketing, v.18, p. 560-578, 2001.

CHENG, B., IOANNOU I.; SERAFEIM G. Corporate Social Responsibility And Access To Finance. Strategic Management Journal v.35, n. 1, p1-23, 2014.

CRANE, A., McWILliamS, A., MATTEN, D., MOON, J.; SIEGEL, D. The Oxford Handbook of Corporate Social Responsibility. New York: The Oxford University Press, 2008. CRAWFORD, F.; MATHEWS R. The Myth of Excellence: Why Great Companies Never Try to Be the Best at Everything. New York: Crown Business, 2001. 
CREYER, E. H.; ROSS, W. T. The Influence of Firm Behavior on Purchase Intention: Do Consumer Really Care About Business Ethics? Journal of Consumer Marketing, v.14, n.6, p.421-433, 1997.

DU, S.; BHATTACHARYA, C.B.; SEN, S.. Reaping relationship rewards from corporate social responsibility: the role of competitive positioning. International Journal of Research in Marketing, v.24, p. 224-241, 2007.

FERREIRA, D. A., ÁVILA, M.; FARIA, M. D. Efeitos da responsabilidade Social corporativa na intenção de compra e no benefício percebido pelo consumidor: um estudo experimental. Revista de Administração (FEA-USP), v.45, n.3, p.285-296, 2010.

FRIEDMAN, M.; FRIEDMAN, R. Capitalism and Freedom. Chicago: University of Chicago Press, 1962.

HAIR, J. F. J.; ANDERSON, R. E.; TATHAM, R. L.; BLACK, W. C. (1998) Multivariate

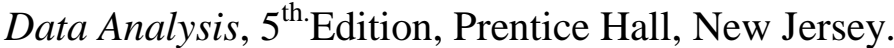

HARRISON, J., e FREEMAN, R. Stakeholders, social responsibility, and performance: Empirical evidence and theoretical perspectives. Academy of Management Journal, v.42, n.5, p.479-485, 1999.

HUBBARB, R.; LINDSAY, R.M. How the emphasis on 'original' empirical marketing research impedes knowledge development. Marketing Theory, Thousand Oaks, v.2, n.4, p.381-402, 2002.

INSTITUTO AKATU e INTITUTO ETHOS (2010) Responsabilidade Social das Empresas Percepção do Consumidor Brasileiro.

ISABELLA, G.; MAZZON, J. A. It Is Not Fair to Pay More! A Study of the Perception of Justice and Price Fairness After Hedonic and Utilitarian Purchases. In: AMA Winter Marketing Educators Conference, 2015, San Antonio, Texas, USA. AMA Winter Marketing Educators Conference, 2015.

KAMAKURA, W. A.; MAZZON, J.A. Socioeconomic Status and Consumpton in an Emerging Economy. International Journal of Research in Marketing. Article in Press, 2013. Disponível em http://dx.doi.org/10.1016/j.ijresmar.2011.12.001_Acesso em Janeiro de 2013.

KLEIN, J.; DAWAR N. Corporate Social Responsibility and Consumers' attributions and Brand Evaluations in a Product-Harm Crisis. International Journal of Research in Marketing, v.21 n.3, p.203-217, 2004.

KONUS, U; VERHOEF, P; NESLIN, S. Multichannel shopper segments and their covariates. Journal of Retailing, v. 84, n. 4, p. 398-413, 2008.

Kraisornsuthasinee, S., \& Swierczek, F. Doing well by doing good in Thailand. Social Responsibility Journal, v. 5, n. 4, p. 550-565, 2009.

LATIN PANEL - Consumidores de Baixa Renda: Onde estão, quem são e o que consomem. www.latinpanel.com.br. Seminário apresentado no GVcev em 17 de Junho de 2004. 
LUO, X.; BHATTACHARYA C. B. Corporate Social Responsibility, Customer Satisfaction, and Market Value. Journal of Marketing, v.70, October, p1-18, 2006.

MASCARENHAS, A. O., DIAS, S. L. G., BAPTISTA, R. M. Elementos para discussão da escravidão contemporânea como prática de gestão. Revista de Administração de Empresas, v.55, n. 2, p. 157-187.

MAXWELL, S. Rule-based price fairness and its effect on willingness to purchase. Journal of Economic Psychology, v.23, p.191-212, 2002

MCGUIRE, J. W. Business and society. New York: McGraw-Hill, 1963.

MOHR, L. A., WEBB, D. J. The Effects of Corporate Social Responsibility and Price on Consumer Responses. The Journal of Consumer Affairs, v.39, n.1, p.121-147, 2005.

NERI, M. C. (2010). A Nova Classe Média: O Lado Brilhante dos Pobres. Centro de Políticas Sociais: FGV, coordenação: Marcelo Cortes Neri. Disponível em: http://www.cps.fgv.br/cps/ncm/. Acesso em novembro de 2012.

NETEMEYER, R.G.; BEARDEN, W. O.; SHARMA, S. Scaling Procedures Issues and Applications. London: Sage Publications, 2003.

PORTER, M.; KRAMER, M. R. A vantagem competitiva da filantropia corporativa. Harvard Business Review - América Latina, v. 80, n.12, p. 42-54, 2002.

PRADO, K. P. L. A. A preferência de marca no processo de decisão de compra: um estudo exploratório no segmento de baixa renda. 2008. Tese (Doutorado em Administração) Faculdade de Economia, Administração e Contabilidade, Universidade de São Paulo, São Paulo, 2008. Disponível em: <http://www.teses.usp.br/teses/disponiveis/12/12139/tde19012009-104931/>. Acesso em: Outubro 2012.

SAEIDI, S. P.; SOFIAN, S.; SAEIDI, P.; SAEIDI, S. P.; SAAEIDI, S. A. How does corporate social responsibility contribute to firm financial performance? The mediating role of competitive advantage, reputation, and customer satisfaction. Journal of Business Research, v.68, n.2, p.341-350, 2015.

SEN, S.; BHATTACHARYA, C. B. Does doing good always lead to doing better? Consumer reactions to corporate social responsibility. Journal of Marketing Research, v.38 n.20, p.225243, 2001.

VAN KENHOVE, P., DE WULF, K., Income and time pressure: a person situation grocery retail typology. International Review of Retail, Distribution \& Consumer Research,v.10 n.2, p.149-166, 2000.

VASCONCELOS, I. F. F. G.; ALVES, M. A.; PESQUEUX, Y. Responsabilidade social corporativa e desenvolvimento sustentável: olhares habermasianos. Revista de Administração de Empresas, v.52, n.2, p.148-152, 2012.

ZEGER, S. L; LIANG, Kung-Yee; ALBERT, Paul S. Models for Longitudinal Data: A Generalized Estimating Equation Approach. Biometrics, v. 44, n.4, p.1049-1060, 1988. 
ZEITHAML, V. A. Consumer Perceptions of Price, Quality, and value: A Means-End Model and Synthesis of Evidence. Journal of Marketing, v.52, n.3, p.2-22, 1988. 